\title{
Fixed points of monotone mappings and application to integral equations
}

\author{
Mostafa Bachar ${ }^{1 *}$ and Mohamed Amine Khamsi ${ }^{2,3}$
}

${ }^{\text {*Correspondence: }}$

mbachar@ksu.edu.sa

'Department of Mathematics, King

Saud University, Riyadh, Saudi

Arabia

Full list of author information is

available at the end of the article

\begin{abstract}
In this work, we discuss the existence of fixed points of monotone nonexpansive mappings defined on partially ordered Banach spaces. This work is a continuity of the previous works of Ran and Reurings, Nieto et al., and Jachimsky done for contraction mappings. As an application, we discuss the existence of solutions to an integral equations.
\end{abstract}

MSC: Primary 46B20; 45D05; secondary 47E10; 34A12

Keywords: fixed point; integral equation; Krasnoselskii iteration; Lebesgue measure; monotone mapping; nonexpansive mapping

\section{Introduction}

Banach's contraction principle [1] is remarkable in its simplicity, yet it is perhaps the most widely applied fixed point theorem in all of analysis. This is because the contractive condition on the mapping is simple and easy to test, because it requires only a complete metric space for its setting, and because it finds almost canonical applications in the theory of differential and integral equations. Over the years, many mathematicians tried successfully to extend this fundamental theorem. Recently a version of this theorem has been given in partially ordered metric spaces $[2,3]$ (see also $[4,5]$ ) and in metric spaces with a graph [6].

In this work, we discuss the case of nonexpansive mappings defined in partially ordered Banach spaces. Nonexpansive mappings are those which have Lipschitz constant equal to 1 . The fixed point theory for such mappings is rich and varied. It finds many applications in nonlinear functional analysis [7]. It is worth mentioning that such investigation is new and has never been carried.

\section{Monotone nonexpansive mappings}

Let $(X,\|\cdot\|)$ be a Banach vector space. Assume that we have a partial order $\preceq$ defined on $X$ such that order intervals are convex and $\tau$-closed, where $\tau$ is a Hausdorff topology on $X$. Recall that an order interval is any of the subsets $[a, b]=\{x \in X ; a \preceq x \preceq b\},[a, \rightarrow)=\{x \in$ $X ; a \preceq x\},(\leftarrow, a]=\{x \in X ; x \preceq a\}$ for any $a, b \in X$.

Definition 2.1 Let $C$ be a nonempty subset of $X$. Let $T: C \rightarrow C$ be a map.

(1) $T$ is said to be monotone if $T(x) \preceq T(y)$ whenever $x \preceq y$ for any $x, y \in C$.

(2) $T$ is said to be monotone nonexpansive if and only if $T$ is monotone and

$$
\|T(x)-T(y)\| \leq\|x-y\|, \quad \text { whenever } x \preceq y .
$$

(c) 2015 Bachar and Khamsi. This article is distributed under the terms of the Creative Commons Attribution 4.0 International License (http://creativecommons.org/licenses/by/4.0/), which permits unrestricted use, distribution, and reproduction in any medium, provided you give appropriate credit to the original author(s) and the source, provide a link to the Creative Commons license, and indicate if changes were made. 
The point $x \in C$ is called a fixed point of $T$ if $T(x)=x$. The set of fixed points of $T$ will be denoted by $\operatorname{Fix}(T)$.

Throughout the paper we assume that $C$ is convex and bounded not reduced to one point. Let $T: C \rightarrow C$ be a monotone nonexpansive mapping. Fix $\lambda \in(0,1)$ and $x_{0} \in C$. The Krasnoselskii $[8,9]$ iteration sequence $\left\{x_{n}\right\} \subset C$ is defined by

$$
x_{n+1}=\lambda x_{n}+(1-\lambda) T\left(x_{n}\right), \quad n \geq 0 .
$$

The following lemma holds.

Lemma 2.1 Under the above assumptions, if we assume that $x_{0} \preceq T\left(x_{0}\right)$, then we have

$$
x_{n} \preceq x_{n+1} \preceq T\left(x_{n}\right) \preceq T\left(x_{n+1}\right)
$$

for any $n \geq 0$. Moreover, if $\left\{x_{n}\right\}$ has two subsequences which $\tau$-converge to $z$ and $w$ respectively, then we must have $z=w$.

Proof First note that if $x \preceq y$ holds, then we have $x \preceq \lambda x+(1-\lambda) y \preceq y$ for any $x, y \in X$ since order intervals are convex. Therefore it is enough to only prove $x_{n} \preceq T\left(x_{n}\right)$ for any $n \geq 0$. By assumption, we have $x_{0} \preceq T\left(x_{0}\right)$. Assume that $x_{n} \preceq T\left(x_{n}\right)$ for $n \geq 1$. Then we have $x_{n} \preceq \lambda x_{n}+(1-\lambda) T\left(x_{n}\right) \preceq T\left(x_{n}\right)$, i.e., $x_{n} \preceq x_{n+1} \preceq T\left(x_{n}\right)$. Since $T$ is monotone, we get $T\left(x_{n}\right) \preceq T\left(x_{n+1}\right)$. By induction, we conclude that the inequalities (KI) hold for any $n \geq 0$. Next let $\left\{x_{\phi(n)}\right\}$ be a subsequence of $\left\{x_{n}\right\}$ which $\tau$-converges to $z$. Clearly, $\left\{\left[x_{n}, \rightarrow\right) ; n \in\right.$ $\mathbb{N}\}$ is a decreasing family of sets. Consequently, if $U_{z} \in \tau$ is a neighborhood of $z$, then $U_{z} \cap\left[x_{n}, \rightarrow\right) \neq \emptyset$ for any $n \in \mathbb{N}$. Therefore, $z$ belongs to all sets $\left[x_{n}, \rightarrow\right)$ as they are closed. Let $w$ be the $\tau$-limit of another subsequence of $\left\{x_{n}\right\}$. If $U_{w} \in \tau$ is a neighborhood of $w$, then $U_{w}$ contains many points from the sequence $\left\{x_{n}\right\}$ since $w$ is a $\tau$-limit of one of its subsequences. Hence $U_{w} \cap(\leftarrow, z] \neq \emptyset$. Therefore, $w$ belongs to $(\leftarrow, z]$ as it is closed, i.e., $w \preceq z$. By reversing the roles of $z$ and $w$, we get $z \preceq w$. The properties of the partial order will force $z=w$ as claimed.

Remark 2.1 Note that under the assumptions of Lemma 2.1, if we assume $T\left(x_{0}\right) \preceq x_{0}$, then we will have

$$
T\left(x_{n+1}\right) \preceq T\left(x_{n}\right) \preceq x_{n+1} \preceq x_{n}
$$

for any $n \geq 0$. The conclusion on the $\tau$-convergence limits of $\left\{x_{n}\right\}$ will also hold.

The following result is found in $[10,11]$.

Proposition 2.1 Under the above assumptions, we have

$$
\begin{aligned}
& (1+n(1-\lambda))\left\|T\left(x_{i}\right)-x_{i}\right\| \\
& \quad \leq\left\|T\left(x_{i+n}\right)-x_{i}\right\|+\lambda^{-n}\left(\left\|T\left(x_{i}\right)-x_{i}\right\|-\left\|T\left(x_{i+n}\right)-x_{i+n}\right\|\right)
\end{aligned}
$$


for any $i, n \in \mathbb{N}$. This inequality implies

$$
\lim _{n \rightarrow+\infty}\left\|x_{n}-T\left(x_{n}\right)\right\|=0
$$

Proof The first part of this proposition is easy to prove via an induction argument on the index $i$. As for the second part, note that $\left\{\left\|x_{n}-T\left(x_{n}\right)\right\|\right\}$ is decreasing. Indeed we have $x_{n+1}-x_{n}=(1-\lambda)\left(T\left(x_{n}\right)-x_{n}\right)$ for any $n \geq 1$. Therefore $\left\{\left\|x_{n}-T\left(x_{n}\right)\right\|\right\}$ is decreasing if and only if $\left\{\left\|x_{n+1}-x_{n}\right\|\right\}$ is decreasing, which holds since

$$
\left\|x_{n+2}-x_{n+1}\right\| \leq \lambda\left\|x_{n+1}-x_{n}\right\|+(1-\lambda)\left\|T\left(x_{n+1}\right)-T\left(x_{n}\right)\right\| \leq\left\|x_{n+1}-x_{n}\right\|
$$

for any $n \geq 0$. Set $\lim _{n \rightarrow+\infty}\left\|x_{n}-T\left(x_{n}\right)\right\|=R$. Then we let $i \rightarrow+\infty$ in the inequality (GK) to obtain

$$
(1+n(1-\lambda)) R \leq \delta(C)
$$

for any $n \in \mathbb{N}$, where $\delta(C)=\sup \{\|x-y\|, x, y \in C\}<+\infty$. Hence

$$
R \leq \frac{\delta(C)}{(1+n(1-\lambda))}, \quad n=1,2, \ldots
$$

which implies $R=0$, i.e., $\lim _{n \rightarrow+\infty}\left\|x_{n}-T\left(x_{n}\right)\right\|=0$.

Before we state the main result of this work, let us recall the definition of Opial condition [12].

Definition 2.2 $X$ is said to satisfy the $\tau$-Opial condition if for any sequence $\left\{y_{n}\right\}$ in $X$ which $\tau$-converges to $y$, we have

$$
\limsup _{n \rightarrow+\infty}\left\|y_{n}-y\right\|<\limsup _{n \rightarrow+\infty}\left\|y_{n}-z\right\|
$$

for any $z \in X$ such that $z \neq y$.

Now we are ready to state the main result of this section.

Theorem 2.1 Let X be a Banach space. Let $\tau$ be a topology on $X$ such that $X$ satisfies the $\tau$-Opial condition. Let $\preceq$ be a partial order on $X$ such that order intervals are convex and $\tau$-closed. Let $C$ be a bounded convex $\tau$-compact nonempty subset of $X$. Let $T: C \rightarrow C$ be a monotone nonexpansive mapping. Assume that there exists $x_{0} \in C$ such that $x_{0}$ and $T\left(x_{0}\right)$ are comparable. Then $T$ has a fixed point.

Proof Without loss of any generality, we assume that $x_{0} \preceq T\left(x_{0}\right)$. Consider the (KIS) sequence $\left\{x_{n}\right\}$ which starts at $x_{0}$. Since $C$ is $\tau$-compact, then $\left\{x_{n}\right\}$ will have a subsequence $\left\{x_{k_{n}}\right\}$ which $\tau$-converges to some point $w \in C$. Lemma 2.1 implies that $\left\{x_{n}\right\} \tau$-converges to $w$ and $x_{n} \preceq w$ for any $n \in \mathbb{N}$. Consider the type function

$$
r(x)=\limsup _{n \rightarrow+\infty}\left\|x_{n}-x\right\|, \quad x \in C .
$$


Then Proposition 2.1 implies $r(x)=\limsup _{n \rightarrow+\infty}\left\|T\left(x_{n}\right)-x\right\|$ for any $x \in C$. Since $T$ is monotone nonexpansive, we get

$$
r(T(w))=\limsup _{n \rightarrow+\infty}\left\|T\left(x_{n}\right)-T(w)\right\| \leq \limsup _{n \rightarrow+\infty}\left\|x_{n}-w\right\|=r(w)
$$

In fact we have $r(T(x)) \leq r(x)$ for any $x \in C$ such that $x_{n}$ and $x$ are comparable for any $n \in \mathbb{N}$. Finally, if $X$ satisfies the $\tau$-Opial condition, then we must have $T(w)=w$, i.e., $w$ is a fixed point of $T$.

The following results are direct consequences of Theorem 2.1.

Corollary 2.1 Let $C$ be a bounded closed convex nonempty subset of $l_{p}, 1<p<+\infty$. Let $\tau$ be the weak topology. Consider the pointwise partial ordering in $l_{p}$, i.e., $\left(\alpha_{n}\right) \preceq\left(\beta_{n}\right)$ iff $\alpha_{n} \leq \beta_{n}$ for all $n \geq 1$. Then any monotone nonexpansive mapping $T: C \rightarrow C$ has a fixed point provided there exists a point $x_{0} \in C$ such that $x_{0}$ and $T\left(x_{0}\right)$ are comparable.

Remark 2.2 The case of $p=1$ is not interesting for the weak topology since $l_{1}$ is a Schur Banach space. But if we consider the weak* topology $\sigma\left(l_{1}, c_{0}\right)$ on $l_{1}$ or the pointwise convergence topology, then $l_{1}$ satisfies the Opial condition for these topologies. Note that these two topologies are Hausdorff. In this case we have a similar conclusion of Corollary 2.1 for $l_{1}$.

Recall the definitions of $l_{p}$ and $c_{0}$ spaces:

(i) $l_{p}=\left\{\left(\alpha_{n}\right) \in \mathbb{R}^{\mathbb{N}}, \sum_{n}\left|\alpha_{n}\right|^{p}<+\infty\right\}$ for $1 \leq p<+\infty$;

(ii) $c_{0}=\left\{\left(\alpha_{n}\right) \in \mathbb{R}^{\mathbb{N}}, \lim _{n \rightarrow+\infty} \alpha_{n}=0\right\}$.

\section{Application to integral equations}

Let us consider the following integral equation of the form

$$
x(t)=g(t)+\int_{0}^{1} F(t, s, x(s)) d s, \quad t \in[0,1]
$$

where

(i) $g$ is in $L^{2}([0,1], \mathbb{R})$,

(ii) $F:[0,1] \times[0,1] \times L^{2}([0,1], \mathbb{R}) \rightarrow \mathbb{R}$ is measurable and satisfies the condition

$$
0 \leq F(t, s, x)-F(t, s, y) \leq x-y
$$

where $t, s \in[0,1]$, and $x, y \in L^{2}([0,1], \mathbb{R})$ such that $y \leq x$.

Recall that for any $u, v \in L^{2}([0,1], \mathbb{R})$, we have

$$
u \leq v \quad \Longleftrightarrow \quad u(t) \leq v(t) \quad \text { almost everywhere } t \in[0,1] .
$$

Condition (3.1) represents the monotonicity of the flow of the integral equation. A comprehensive study of the monotonicity of the flow can be found in the book of Smith [13]. 
Assume that there exists a non-negative function $h(\cdot, \cdot) \in L^{2}([0,1] \times[0,1])$ and $M<\frac{1}{2}$ such that

$$
|F(t, s, x)| \leq h(t, s)+M|x|
$$

where $t, s \in[0,1]$ and $x \in L^{2}([0,1], \mathbb{R})$.

Let

$$
B=\left\{y \in L^{2}([0,1], \mathbb{R}) \text {, such that }\|x\|_{L^{2}([0,1], \mathbb{R})} \leq \rho\right\},
$$

where $\rho$ is sufficiently large, i.e., $B$ is the closed ball of $L^{2}([0,1], \mathbb{R})$ centered at 0 with radius $\rho$. Consider the operator defined by

$$
\widetilde{F}(t)(y)(s)=F(t, s, y(s)),
$$

and define the operator $J: L^{2}([0,1], \mathbb{R}) \rightarrow L^{2}([0,1], \mathbb{R})$ by

$$
(J y)(t)=g(t)+\int_{0}^{1} \widetilde{F}(t)(y)(s) d s .
$$

We have $J(B) \subset B$. Indeed let $x \in B$, then by using the Cauchy-Schwarz inequality, condition (3.2) and the quadratic inequality $(a+b)^{2} \leq 2 a^{2}+2 b^{2}$ for any $a, b \in \mathbb{R}$, we have

$$
\begin{aligned}
\|J x\|_{L^{2}([0,1], \mathbb{R})}^{2} & =\int_{0}^{1}|J x(t)|^{2} d t \\
& =\int_{0}^{1}\left|g(t)+\int_{0}^{1} \widetilde{F}(t)(x)(s) d s\right|^{2} d t \\
& \leq 2 \int_{0}^{1}|g(t)|^{2} d t+2 \int_{0}^{1} \int_{0}^{1}|\widetilde{F}(t)(x)(s)|^{2} d s d t \\
& \leq 2 \int_{0}^{1}|g(t)|^{2} d t+\left.2 \int_{0}^{1} \int_{0}^{1}|h(t, s)+M| x(s)\right|^{2} d s d t \\
& \leq 2 \int_{0}^{1}|g(t)|^{2} d t+4 \int_{0}^{1} \int_{0}^{1}|h(t, s)|^{2} d s d t+4 M^{2} \int_{0}^{1} \int_{0}^{1}|x(s)|^{2} d s d t \\
& =2 \int_{0}^{1}|g(t)|^{2} d t+4 \int_{0}^{1} \int_{0}^{1}|h(t, s)|^{2} d s d t+4 M^{2} \int_{0}^{1}|x(s)|^{2} d s \\
& =2 \int_{0}^{1}|g(t)|^{2} d t+4 \int_{0}^{1} \int_{0}^{1}|h(t, s)|^{2} d s d t+4 M^{2}\|x\|_{L^{2}([0,1], \mathbb{R})}^{2} \\
& \leq 2 \int_{0}^{1}|g(t)|^{2} d t+4 \int_{0}^{1} \int_{0}^{1} h^{2}(t, s) d s d t+4 M^{2} \rho^{2} .
\end{aligned}
$$

Since $M<1 / 2$, choose $\rho$ such that

$$
\frac{2}{\left(1-4 M^{2}\right)} \int_{0}^{1}|g(t)|^{2} d t+\frac{4}{\left(1-4 M^{2}\right)} \int_{0}^{1} \int_{0}^{1} h^{2}(t, s) d s d t \leq \rho^{2},
$$

we will get $J(x) \in B$ as claimed. Next we prove that $J$ is monotone nonexpansive. First from condition (3.1), $J$ is obviously monotone. Let $x, y \in L^{2}([0,1], \mathbb{R})$ such that $y \leq x$. Using the 
Cauchy-Schwarz inequality, we have

$$
\begin{aligned}
\|J x-J y\|_{L^{2}([0,1], \mathbb{R})}^{2} & =\int_{0}^{1}(J x(t)-J y(t))^{2} d t \\
& =\int_{0}^{1}\left(\int_{0}^{1}(\widetilde{F}(t)(x)(s)-\widetilde{F}(t)(y)(s)) d s\right)^{2} d t \\
& \leq \int_{0}^{1}\left(\int_{0}^{1}(x(s)-y(s)) d s\right)^{2} d t \\
& \leq \int_{0}^{1}(x(s)-y(s))^{2} d s=\|x-y\|_{L^{2}([0,1], \mathbb{R})}^{2}
\end{aligned}
$$

which implies that $J$ is a monotone nonexpansive operator as claimed. In order to use Theorem 2.1, we need to check its assumptions. First note that $X=L^{2}([0,1], \mathbb{R})$ is a Hilbert space. If we choose $\tau$ to be the weak topology, then $X$ satisfies the weak Opial condition. It is easy to check that order intervals are convex. In order to show that order intervals are closed, we will show that if $\left\{u_{n}\right\}$ is a non-negative sequence of elements in $X$ which converges weakly to $u$, then $u$ is positive. Let $a<0$, then the set $A=\{t \in[0,1] ; u(t) \leq a\}$ has measure 0 . Indeed, we have

$$
\int_{A} u(t) d t=\lim _{n \rightarrow+\infty} \int_{A} u_{n}(t) d t
$$

because of weak convergence. So

$$
0 \leq \lim _{n \rightarrow+\infty} \int_{A} u_{n}(t) d t=\int_{A} u(t) d t \leq \operatorname{am}(A) \leq 0
$$

Hence $m(A)=0$. Set

$$
D=\bigcup_{n \geq 1}\left\{t \in[0,1] ; u(t) \leq-\frac{1}{n}\right\}
$$

Then $D$ has measure 0 , which implies that $u(t) \geq 0$ for almost every $t \in[0,1]$. Using Theorem 2.1, we get the following result.

Theorem 3.1 Under the above assumptions, we conclude that

(i) the integral equation (IE) has a non-negative solution provided we assume that $g(t)+\int_{0}^{1} F(t, s, 0) d s \geq 0$ for almost every $t \in[0,1]$ (which implies $J(0) \geq 0$ );

(ii) the integral equation (IE) has a non-positive solution provided we assume that $g(t)+\int_{0}^{1} F(t, s, 0) d s \leq 0$ for almost every $t \in[0,1]$ (which implies $\left.J(0) \leq 0\right)$.

The authors declare that they have no competing interests. 


\section{Acknowledgements}

The authors would like to extend their sincere appreciation to the Deanship of Scientific Research at King Saud University for funding this Research group No. (RG-1435-079).

Received: 27 February 2015 Accepted: 23 June 2015 Published online: 10 July 2015

\section{References}

1. Banach, S: Sur les opérations dans les ensembles abstraits et leur application. Fundam. Math. 3, 133-181 (1922)

2. Nieto, JJ, Rodríguez-López, R: Contractive mapping theorems in partially ordered sets and applications to ordinary differential equations. Order 22, 223-239 (2005)

3. Ran, ACM, Reurings, MCB: A fixed point theorem in partially ordered sets and some applications to matrix equations. Proc. Am. Math. Soc. 132, 1435-1443 (2004)

4. Turinici, M: Fixed points for monotone iteratively local contractions. Demonstr. Math. 19, 171-180 (1986)

5. Turinici, M: Ran and Reurings theorems in ordered metric spaces. J. Indian Math. Soc. 78, 207-2014 (2011)

6. Jachymski, J: The contraction principle for mappings on a metric space with a graph. Proc. Am. Math. Soc. 136 1359-1373 (2008)

7. Browder, FE: Nonexpansive nonlinear operators in a Banach space. Proc. Natl. Acad. Sci. USA 54, 1041-1044 (1965)

8. Ishikawa, S: Fixed points and iteration of a nonexpansive mapping in a Banach space. Proc. Am. Math. Soc. 59, 65-71 (1976)

9. Krasnoselskii, MA: Two observations about the method of successive approximations. Usp. Mat. Nauk 10, 123-127 (1955)

10. Goebel, K, Kirk, WA: Iteration processes for nonexpansive mappings. Contemp. Math. 21, 115-123 (1983)

11. Goebel, K, Kirk, WA: Topics in Metric Fixed Point Theory. Cambridge Stud. Adv. Math., vol. 28. Cambridge University Press, Cambridge (1990)

12. Opial, Z: Weak convergence of the sequence of successive approximations for nonexpansive mappings. Bull. Am. Math. Soc. 73, 591-597 (1967)

13. Smith, JL: Monotone Dynamical Systems. Mathematical Surveys and Monographs, vol. 41. Am. Math. Soc., Providence (1995)

\section{Submit your manuscript to a SpringerOpen ${ }^{\mathcal{O}}$ journal and benefit from:}

- Convenient online submission

- Rigorous peer review

Immediate publication on acceptance

- Open access: articles freely available online

- High visibility within the field

- Retaining the copyright to your article 